\title{
Impact of Local Congruences in Attribute Reduction
}

\author{
Roberto G. Aragón, Jesús Medina, and Eloísa Ramírez-Poussa $\left.{ }^{(}\right)$ \\ Department of Mathematics, University of Cádiz, Cádiz, Spain \\ \{roberto.aragon, jesus.medina, eloisa.ramirez\}@uca.es
}

\begin{abstract}
Local congruences are equivalence relations whose equivalence classes are convex sublattices of the original lattice. In this paper, we present a study that relates local congruences to attribute reduction in FCA. Specifically, we will analyze the impact in the context of the use of local congruences, when they are used for complementing an attribute reduction.
\end{abstract}

Keywords: Formal Concept Analysis - Size reduction · Attribute reduction · Local congruence

\section{Introduction}

Formal Concept Analysis (FCA) is a mathematical framework to analyze datasets introduced by Ganter and Wille in eighties [12]. The main goals of FCA are the following: to obtain the knowledge from data, to represent the obtained knowledge by means of the mathematical structure called concept lattice and to discover dependencies in data. The applied potential of FCA has encouraged the development of different generalizations.

One of the most intensively studied research lines by the research community of FCA in the last years, consists on decreasing the number of attributes of a dataset, preserving the information provided by the dataset $[1,2,7,8,10,11,13$ 18]. In [6], authors proved that every reduction of attributes of a formal context induces an equivalent relation whose equivalent classes are join-semilattices. In [3], local congruences were introduced and applied to this attribute reduction. Local congruences are equivalence relations on lattices whose equivalence classes are convex sublattices. The idea in [3] was to find the least local congruence containing the equivalent relation induced by an attribute reduction of a formal context, in order to group the concepts of the original concept lattice using closed structures.

Partially supported by the the 2014-2020 ERDF Operational Programme in collaboration with the State Research Agency (AEI) in project TIN2016-76653-P, and with the Department of Economy, Knowledge, Business and University of the Regional Government of Andalusia. in project FEDER-UCA18-108612, and by the European Cooperation in Science \& Technology (COST) Action CA17124.

(C) Springer Nature Switzerland AG 2020

M.-J. Lesot et al. (Eds.): IPMU 2020, CCIS 1239, pp. 748-758, 2020.

https://doi.org/10.1007/978-3-030-50153-2_55 
Sometimes, the induced equivalent relation by a reduction of the context is already a local congruence but sometime it is not. In the latter case, the fact of using a local congruence that contains the induced equivalence relation has an influence on the original reduction. In this paper, we present an initial study about the relationship between local congruences and the induced equivalent relation by an attribute reduction of a formal context. This study provides a first step to know the influence that this special kind of equivalence relations has on the reduction procedure. We will include several examples to illustrate the obtained result.

\section{Preliminaries}

We need to recall some basic notions used in this work. In order to present the preliminary notions as clearly as possible, we will divide this section into two parts. The first one will be devoted to recall those necessary notions of FCA and the second one to those related to local congruences.

\subsection{Formal Concept Analysis}

In FCA a context is a triple $(A, B, R)$ where $A$ is a set of attributes, $B$ is a set of objects and $R: A \times B \rightarrow\{0,1\}$ is a relationship, such that $R(a, x)=a R x=1$, if the object $x \in B$ possesses the attribute $a \in A$, and $R(a, x)=0$, otherwise. In addition, we call concept-forming operators to the mappings $\uparrow: 2^{B} \rightarrow 2^{A}$ and $\downarrow: 2^{A} \rightarrow 2^{B}$ defined for each $X \subseteq B$ and $Y \subseteq A$ as:

$$
\begin{aligned}
& X^{\uparrow}=\{a \in A \mid \text { for all } x \in X, a R x\} \\
& Y^{\downarrow}=\{x \in B \mid \text { for all } a \in Y, a R x\}
\end{aligned}
$$

Taking into account the previous mappings, a concept is a pair $(X, Y)$, with $X \subseteq B$ and $Y \subseteq A$ satisfying that $X^{\uparrow}=Y$ and $Y^{\downarrow}=X$. The subset $X$ is called the extent of the concept and the subset $Y$ is called the intent. The set of extents and intents are denoted by $\mathfrak{E}(A, B, R)$ and $\mathfrak{I}(A, B, R)$, respectively.

In addition, all the concepts together with the inclusion ordering on the left argument has the structure of a complete lattice, which is called concept lattice and it is denoted as $\mathcal{C}(A, B, R)$.

From now on, we will say that an attribute-concept is a concept generated by an attribute $a \in A$, that is $\left(a^{\downarrow}, a^{\downarrow \uparrow}\right)$.

On the other hand, we need to recall the notion of meet-irreducible element of a lattice.

Definition 1. Given a lattice $(L, \preceq)$, such that $\wedge$ is the meet operator, and an element $x \in L$ verifying

1. If $L$ has a top element $\top$, then $x \neq \top$.

2. If $x=y \wedge z$, then $x=y$ or $x=z$, for all $y, z \in L$. 
we call $x$ meet-irreducible $(\wedge$-irreducible) element of $L$. Condition (2) is equivalent to

$2^{\prime}$. If $x<y$ and $x<z$, then $x<y \wedge z$, for all $y, z \in L$.

On the other hand, with respect to the attribute reduction in FCA, it is important to recall that when we reduce the set of attributes in a context, an equivalence relation on the set of concepts of the original concept lattice is induced. The following proposition was proved in [6] for the classical setting of FCA and it is recalled below.

Proposition 1 ([6]). Given a context $(A, B, R)$ and a subset $D \subseteq A$. The set $R_{E}=\left\{\left(\left(X_{1}, Y_{1}\right),\left(X_{2}, Y_{2}\right)\right) \mid\left(X_{1}, Y_{1}\right),\left(X_{2}, Y_{2}\right) \in \mathcal{C}(A, B, R), X_{1}^{\uparrow D \downarrow}=X_{2}^{\uparrow D \downarrow}\right\}$ is an equivalence relation. Where ${ }^{\uparrow D}$ denotes the concept-forming operator $X^{\uparrow_{D}}=\{a \in D \mid$ for all $x \in X,(a, x) \in R\}$ restricted to the subset of attributes $D \subseteq A$.

In [6], the authors also proved that each equivalence class of the induced equivalence relation has a structure of join semilattice.

Proposition 2 ([6]). Given a context $(A, B, R)$, a subset $D \subseteq A$ and a class $[(X, Y)]_{D}$ of the quotient set $\mathcal{C}(A, B, R) / R_{E}$. The class $[(X, Y)]_{D}$ is a join semilattice with maximum element $\left(X^{\uparrow} \downarrow \downarrow, X^{\uparrow} \downarrow \uparrow\right)$.

\subsection{Local Congruences}

The notion of local congruence arose with the goal of complementing attribute reduction in FCA. The purpose of local congruences is to obtain equivalence relations less-constraining than congruences [3] and with useful properties to be applied in size reduction processes of concept lattices. We recall the notion of local congruence in the next definition.

Definition 2. Given a lattice $(L, \preceq)$, we say that an equivalence relation $\delta$ on $L$ is a local congruence if each equivalence class of $\delta$ is a convex sublattice of $L$.

The notion of local congruence can be characterized in terms of the equivalence relation, as the following result shows.

Proposition 3. Given a lattice $(L, \preceq)$ and an equivalence relation $\delta$ on $L$, the relation $\delta$ is a local congruence on $L$ if and only if, for each $a, b, c \in L$, the following properties hold:

(i) If $(a, b) \in \delta$ and $a \preceq c \preceq b$, then $(a, c) \in \delta$.

(ii) $(a, b) \in \delta$ if and only if $(a \wedge b, a \vee b) \in \delta$.

Usually, we will look for a local congruence that contains a partition induced by an equivalence relation. When we say that a local congruence contain a partition provided by an equivalence relation, we are making use of the following definition of inclusion of equivalence relations.

Definition 3. Let $\rho_{1}$ and $\rho_{2}$ be two equivalence relations on a lattice $(L, \preceq)$. We say that the equivalence relation $\rho_{1}$ is included in $\rho_{2}$, denoted as $\rho_{1} \sqsubseteq \rho_{2}$, if for every equivalence class $[x]_{\rho_{1}} \in L / \rho_{1}$ there exists an equivalence class $[y]_{\rho_{2}} \in L / \rho_{2}$ such that $[x]_{\rho_{1}} \subseteq[y]_{\rho_{2}}$. 


\section{Analyzing Local Congruences}

In this section, we will present an initial study about the role of local congruences when they are used along or together with other mechanisms to attribute reduction. In particular, we will analyze the relationship between local congruences and the induced equivalence relation by an attribute reduction from the perspective of the attribute of the context as well as from the meet-irreducible elements of the concept lattices. We are interested in discovering under what conditions the induced equivalence relation is a local congruence. We are also interested in analyzing the influence of the use of local congruence in the reduction of attributes, when the induced equivalence relation is not a local congruence.

Firstly, in the first example we will illustrate the main idea of this study.

Example 1. Let us consider a formal context $(A, B, R)$ composed of the attributes $A=\left\{a_{1}, a_{2}, a_{3}\right\}$ and the objects $B=\left\{b_{1}, b_{2}, b_{3}\right\}$, related by a relationship $R \subseteq A \times B$, which is shown in the left side of Table 1, together with the list of concepts which appears in the right side of the same table. The associated concept lattice is displayed in the left side of Fig. 1.

Table 1. Relation and list of concepts of the context of Example 1.

\begin{tabular}{c|ccc}
\hline$R$ & $b_{1}$ & $b_{2}$ & $b_{3}$ \\
\hline$a_{1}$ & 1 & 0 & 1 \\
$a_{2}$ & 0 & 1 & 1 \\
$a_{3}$ & 0 & 0 & 1 \\
\hline
\end{tabular}

\begin{tabular}{cccccccc}
\hline$C_{i}$ & \multicolumn{2}{l}{ Extent } & & \multicolumn{3}{l}{ Intent } \\
\cline { 2 - 3 } \cline { 6 - 8 } & $b_{1}$ & $b_{2}$ & $b_{3}$ & & $a_{1}$ & $a_{2}$ & $a_{3}$ \\
\hline 0 & 0 & 0 & 1 & 1 & 1 & 1 \\
1 & 1 & 0 & 1 & 1 & 0 & 0 \\
2 & 0 & 1 & 1 & 0 & 1 & 0 \\
3 & 1 & 1 & 1 & 0 & 0 & 0 \\
\hline
\end{tabular}

In order to analyze the influence of local congruences in the reduction of the set of attributes of the considered context, we include a list in which we show the attribute that generates each concept of the concept lattice:

$$
\begin{aligned}
& C_{0}=\left(a_{3}^{\downarrow}, a_{3}^{\downarrow \uparrow}\right) \\
& C_{1}=\left(a_{1}^{\downarrow}, a_{1}^{\downarrow \uparrow}\right) \\
& C_{2}=\left(a_{2}^{\downarrow}, a_{2}^{\downarrow \uparrow}\right)
\end{aligned}
$$

If we consider, for example, the subset $D_{1}=\left\{a_{2}, a_{3}\right\}$ to carry out the reduction of the set of attributes, that is, we remove the attribute $a_{1}$, we obtain a partition of the concept lattice induced by the reduction that is highlighted by means of a dashed Venn diagram in the middle of Fig. 1. We obtain that the concepts $C_{1}$ and $C_{3}$ are grouped in the same class whereas the concepts $C_{0}$ and $C_{2}$ provide two different classes composed of a single concept each one. Therefore, according to Proposition 2, we can see that the obtained equivalence classes are 

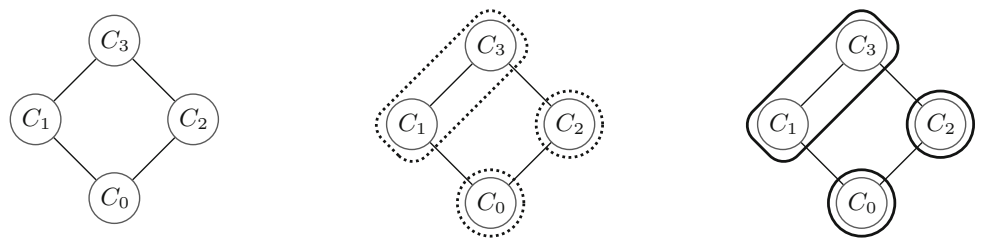

Fig. 1. Concept lattice of Example 1 (left), the partition induced by the subset $D_{1}$ (center) and the least local congruence containing the partition (right).

join semilattices. Indeed, all classes are convex sublattices of the original concept lattice.

As a consequence, the least local congruence containing such a reduction is the induced partition itself as it is shown in the right side of Fig. 1, where the local congruence is highlighted by means of a Venn diagram. In other words, the induced equivalence relation by the reduction is already a local congruence and, as a consequence, the consideration of local congruences does not alter the attribute reduction originally carried out on the set of attributes.

However, if the user decides to remove the attributes $a_{1}$ and $a_{2}$, that is, only the subset of attributes $D_{2}=\left\{a_{3}\right\}$ is considered, the induced partition by the reduction is shown in the left side of Fig. 2.
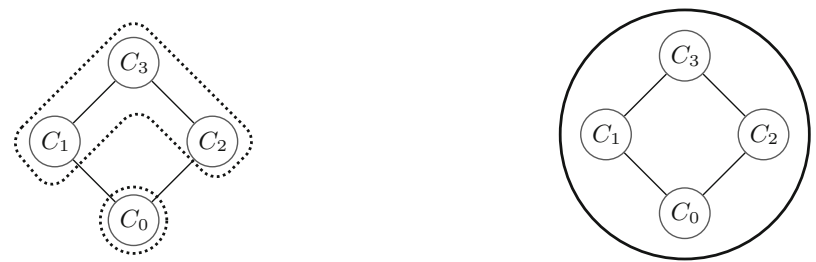

Fig. 2. The partition induced by the elimination of the attributes $a_{1}$ and $a_{2}$ of Example 1 (left) and the least local congruence containing the induced partition (right).

The equivalence classes induced by the the reduction are the following:

$$
\begin{aligned}
& {\left[C_{0}\right]_{D_{2}} }=\left\{C_{0}\right\} \\
& {\left[C_{1}\right]_{D_{2}}=\left[C_{2}\right]_{D_{2}}=\left[C_{3}\right]_{D_{2}}=\left\{C_{1}, C_{2}, C_{3}\right\} }
\end{aligned}
$$

In this case, the obtained equivalence classes are non-trivial join-semilattices since the concepts $C_{1}, C_{2}$ and $C_{3}$ do not form a convex sublattice of the original concept lattice. In this case, the infimum of the equivalence class $\left[C_{1}\right]_{D_{2}}$ is de concept $C_{0}$, which has not been included in $\left[C_{1}\right]_{D_{2}}$. This concept is not in $\left[C_{1}\right]_{D_{2}}$ because it is generated from the attribute $a_{3}$, which means that this attribute differences concept $C_{0}$ from the rest. Therefore, if this attribute is not removed in the reduction procedure, then it continues differentiating this concept from the rest and it cannot be in the same class of the rest. 
If we compute the least local congruence containing the equivalence relation above, it groups all concepts in a single class, that is, the local congruence includes the infimum of the concepts $C_{1}, C_{2}$ and $C_{3}$, that is, the concept $C_{0}$, in the equivalence class $\left[C_{1}\right]_{D_{2}}$. This local congruence is depicted in the right side of Fig. 2. Clearly, this local congruence does not coincides with the equivalence relation induced by the attribute reduction which entails certain consequences with respect to the initial attribute reduction, since the inclusion of the concept $C_{0}$ in the equivalence class $\left[C_{1}\right]_{D_{2}}$, can be seen as a kind of elimination of the attribute $a_{3}$ (since the attribute $a_{3}$ generates the concept $C_{0}$ ).

The previous example has shown different possibilities of applying local congruences for complementing an attribute reduction process. Hence, we have that some times the obtained equivalence relations is already a local congruence and other cases is not. In particular, we have seen a case that when the infimum of an induced equivalence class is generated by an attribute, which has not been removed during the reduction process, proper join semilattices arise and the induced equivalence relation is not a local congruence. In the following example, we will analyze another possible situations we can find when the set of attributes is reduced.

Example 2. We will consider a context composed of the set of attributes $A=$ $\left\{a_{1}, a_{2}, a_{3}, a_{4}\right\}$ and the set of objects $B=\left\{b_{1}, b_{2}, b_{3}\right\}$, related by $R: A \times B \rightarrow$ $\{0,1\}$, defined on the left side of Table 2 together with the list of the corresponding concepts which appear in the right side of the same table. The associated concept lattice is given on the left side of Fig. 3 .

Table 2. Relation and list of concepts of the context of Example 2.

\begin{tabular}{c|ccc}
\hline$R$ & $b_{1}$ & $b_{2}$ & $b_{3}$ \\
\hline$a_{1}$ & 1 & 1 & 0 \\
$a_{2}$ & 1 & 0 & 1 \\
$a_{3}$ & 0 & 1 & 1 \\
$a_{4}$ & 0 & 0 & 1 \\
\hline
\end{tabular}

\begin{tabular}{|c|c|c|c|c|c|c|c|}
\hline \multirow[t]{2}{*}{$C_{i}$} & \multicolumn{3}{|c|}{ Extent } & \multicolumn{4}{|c|}{ Intent } \\
\hline & $b_{1}$ & $b_{2}$ & $b_{3}$ & $a_{1}$ & $a_{2}$ & $a_{3}$ & $a_{4}$ \\
\hline 0 & 0 & 0 & 0 & 1 & 1 & 1 & 1 \\
\hline 1 & 1 & 0 & 0 & 1 & 1 & 0 & 0 \\
\hline 2 & 0 & 1 & 0 & 1 & 0 & 1 & 0 \\
\hline 3 & 0 & 0 & 1 & 0 & 1 & 1 & 1 \\
\hline 4 & 1 & 1 & 0 & 1 & 0 & 0 & 0 \\
\hline 5 & 1 & 0 & 1 & 0 & 1 & 0 & 0 \\
\hline 6 & 0 & 1 & 1 & 0 & 0 & 1 & 0 \\
\hline 7 & 1 & 1 & 1 & 0 & 0 & 0 & 0 \\
\hline
\end{tabular}


From this context we obtain the following attribute-concepts:

$$
\begin{aligned}
& C_{3}=\left(a_{4}^{\downarrow}, a_{4}^{\downarrow \uparrow}\right) \\
& C_{4}=\left(a_{1}^{\downarrow}, a_{1}^{\downarrow \uparrow}\right) \\
& C_{5}=\left(a_{2}^{\downarrow}, a_{2}^{\downarrow \uparrow}\right) \\
& C_{6}=\left(a_{3}^{\downarrow}, a_{3}^{\downarrow \uparrow}\right)
\end{aligned}
$$

For instance, if we are interested in considering the subset of attributes $D_{1}=$ $\left\{a_{1}, a_{3}\right\}$ and we carry out the corresponding reduction (removing the attributes $a_{2}$ and $a_{4}$ ), we obtain the partition induced by $D_{1}$, which is shown in the middle of Fig. 3. Once again, as in Example 1, the equivalence classes obtained from the reduction considering the subset $D_{1}$ are convex sublattices of the original concept lattice. Therefore, the least local congruence that contains such a reduction is the induced equivalence relation itself, as it can be seen in the right side of Fig. 3. Consequently, local congruences do not modify the considered attribute reduction.
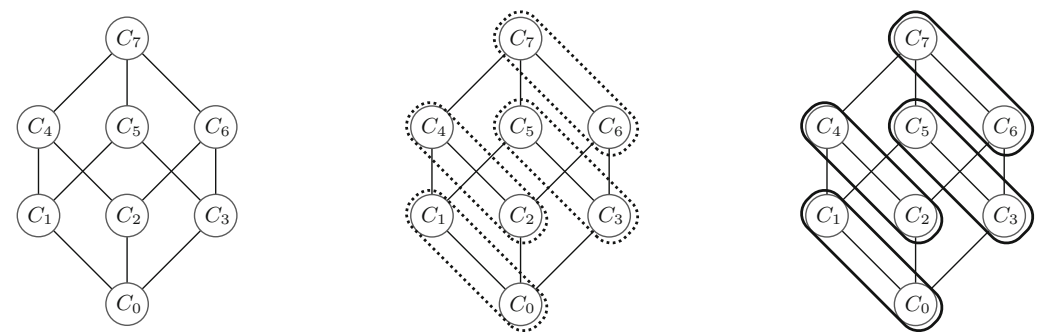

Fig. 3. Concept lattice of Example 2 (left), the partition induced by the subset $D_{1}$ (center) and the least local congruence containing the induced partition (right).

Now, if the attributes $a_{2}$ and $a_{3}$ are removed, i.e., only the subset of attributes $D_{2}=\left\{a_{1}, a_{4}\right\}$ is considered, then the partition induced by the reduction is shown in the left side of Fig. 4 and the induced equivalence classes are listed below.

$$
\begin{aligned}
& {\left[C_{0}\right]_{D_{2}}=\left\{C_{0}\right\} } \\
& {\left[C_{1}\right]_{D_{2}}=\left[C_{2}\right]_{D_{2}}=} {\left[C_{4}\right]_{D_{2}}=\left\{C_{1}, C_{2}, C_{4}\right\} } \\
& {\left[C_{3}\right]_{D_{2}}=\left\{C_{3}\right\} } \\
& {\left[C_{5}\right]_{D_{2}}=\left[C_{6}\right]_{D_{2}}=\left[C_{7}\right]_{D_{2}}=\left\{C_{5}, C_{6}, C_{7}\right\} }
\end{aligned}
$$

Notice that two of the obtained equivalence classes are not convex sublattices of the original concept lattice. The first one contains the concepts $C_{1}, C_{2}, C_{4}$ and the other one contains the concepts $C_{5}, C_{6}, C_{7}$. However, the reasons that make these classes are not convex sublattices are well differentiated. 
On the one hand, with respect to the equivalence class of the concept $\left[C_{5}\right]_{D_{2}}$ we find a similar situation than the one shown in Example1, that is, the infimum of the equivalence class $\left[C_{5}\right]_{D_{2}}$ is the concept $C_{3}$ which is generated from attribute $a_{4}$ that has not been removed in the reduction of the context.

On the other hand, the infimum of the equivalence class $\left[C_{1}\right]_{D_{2}}$ is the concept $C_{0}$ which is not generated by any attribute of the context. Nevertheless, $C_{0} \notin$ $\left[C_{1}\right]_{D_{2}}$ since in the decomposition of meet-irreducible concepts of the concept $C_{0}$, that is $C_{0}=C_{4} \wedge C_{5} \wedge C_{6}$, we can find two meet-irreducible concepts $C_{5}$ and $C_{6}$ satisfying that $C_{5}, C_{6} \notin\left[C_{1}\right]_{D_{2}}$.

In this case, the least local congruence whose equivalence classes contain the induced partition can be seen in the right side of Fig. 4. In this figure we have that the local congruence includes the infimum of the equivalence classes $\left[C_{1}\right]_{D_{2}}$ and $\left[C_{5}\right]_{D_{2}}$ in their respective classes. Thus, the least local congruence provides two different equivalence classes.
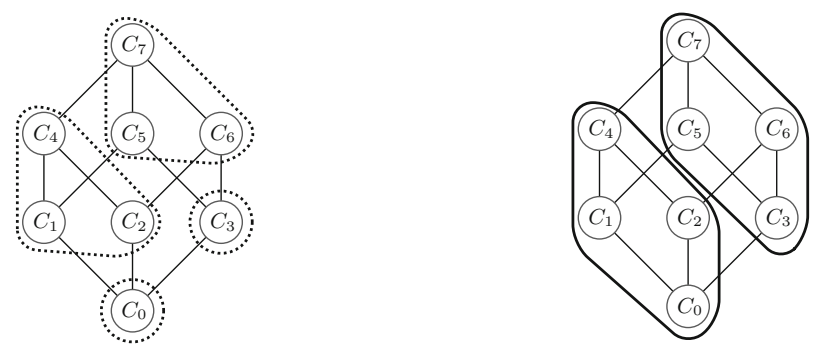

Fig. 4. The partition induced by the elimination of attributes $a_{2}$ and $a_{3}$ in Example 2 (left) and the least local congruence containing the induced partition (right).

Now, we will analyze how this local congruence influences in the reduction of the attributes. We can see that the inclusion of the concept $C_{3}$ in the equivalence $\left[C_{5}\right]_{D_{2}}$, is equivalent to the elimination of attribute $a_{4}$. We can also observe that the intension of the concept $C_{0}$ includes attribute $a_{4}$ which is ignored when $C_{0}$ is introduced in the equivalence class $\left[C_{5}\right]_{D_{2}}$. Hence, in spite of the reduction of the context was carried out originally from the elimination of attributes $a_{2}$ and $a_{3}$, somehow the consideration of the local congruence implies the elimination of attribute $a_{4}$.

From the previous examples, we deduce that when the induced equivalence relation does not provide convex sublattices as equivalence classes, the use of local congruence relations alters the original attribute reduction, increasing the number of attributes to be removed. Moreover, it would be interesting to highlight these attributes, record its relationship with the removed attributes and the impact in attribute implications $[4,5,9,19]$.

Next result relates the equivalence relations induced by an attribute reduction with the attributes-concepts and the meet-irreducible elements of the concept lattice. Due to the closely relation between the $\wedge$-irreducible concepts and the 
set of attributes of the context. This result summarizes the influence of local congruences in the attribute reduction of relational datasets.

Proposition 4. Given a context $(A, B, R)$, a subset of attributes $D \subseteq A$, an equivalence class $[C]_{D}$, with $C \in \mathcal{C}(A, B, R)$, of the induced equivalence relation which is not a convex sublattice and the concept $C^{\prime}=\bigwedge_{C_{i} \in[C]_{D}} C_{i}$. Then, one of the following statements is satisfied:

- There exists at least one attribute $a \in A$ such that $C^{\prime}=\left(a^{\downarrow}, a^{\downarrow \uparrow}\right)$.

- There exists a concept $C^{*} \in M_{F}(A, B, R)$ in a meet-irreducible decomposition $\left\{C_{j} \in M_{F}(A, B, R) \mid j \in J\right\}$ of $C^{\prime}$, such as $C_{i_{0}} \not \leq C^{*}$ for a concept $C_{i_{0}} \in$ $[C]_{D}$.

Proof. Let us assume that we reduce the context $(A, B, R)$, by considering a subset of attributes $D \subseteq A$, and that given $C \in \mathcal{C}(A, B, R)$, the induced equivalence class $[C]_{D}$ is not a convex sublattice of the original concept lattice.

Therefore, although by Proposition 2 the class $[C]_{D}$ is a join-semilattice, the concept $C^{\prime}=\bigwedge_{C_{i} \in[C]_{D}} C_{i}$ is not in $[C]_{D}=\left\{C_{1}, \ldots, C_{n}\right\}$. Now, we will distinguish two cases:

(i) If there exists $a_{0} \in A$ such that $C^{\prime}=\left(a_{0}^{\downarrow}, a_{0}^{\downarrow \uparrow}\right)$, the first statement holds.

(ii) Otherwise, let $\left\{C_{j} \in M_{F}(A, B, R) \mid j \in J\right\}$ be a meet-irreducible decomposition of $C^{\prime}$, that is, $C^{\prime}=\bigwedge_{j \in J} C_{j}$. If there exists $j_{0} \in J$ and $i_{0} \in\{1, \ldots, n\}$, such as $C_{i_{0}} \not \leq C_{j_{0}}$ for all $C_{i} \in[C]_{D}$, we finish the proof. Otherwise, we have that $C_{i} \leq C_{j}$ for all $C_{i} \in[C]_{D}$ and $j \in J$. As a consequence, the set $\left\{C_{j} \in M_{F}(A, B, R) \mid j \in J\right\}$ is in the meet-irreducible decomposition of every concept in $[C]_{D}$, in particular in the maximum element of the class, denoted as $C_{M}$. Hence, we have that

$$
C^{\prime}<C_{M} \leq \bigwedge_{j \in J} C_{j}=C^{\prime}
$$

which leads us to a contradiction.

It is important to mention that the items exposed in the previous result are not exclusive, that is, we can find a concept $C^{\prime}$ satisfying simultaneously both conditions of the previous result. In this situation, this fact means that the intent of the concept $C^{\prime}$ has at least two different attributes, $a_{0}$ and $a_{1}$ such that these attributes do not belong to the intent of any concept $C_{i} \in[C]_{D}$ for all $i \in I$.

Notice also that the requirement " $C_{i_{0}} \not \leq C^{*}$ for a concept $C_{i_{0}} \in[C]_{D}$." in the second condition can be rewritten as $C_{i_{0}}$ and $C^{*}$ are incomparable, or $C^{*}<C_{i_{0}}$. This last inequality detects a possible non-distributivity lattice and discover the following consequences of Proposition 4.

Corollary 1. Let $(A, B, R)$ be a context where its concept lattice $\mathcal{C}(A, B, R)$ is distributive, $D \subseteq A$ a subset of attributes and $C \in M_{F}(A, B, R)$. If $C^{\prime}=$ $\bigwedge_{C_{i} \in[C]_{D}} C_{i}$ is not in $[C]_{D}$, then there exists an attribute $a \in A$ such that $C^{\prime}=$ $\left(a^{\downarrow}, a^{\downarrow \uparrow}\right)$. 
These results show that the application of local congruences offers an advance and complemented procedure to reduce concept lattices, selecting and removing appropriate new attributes.

\section{Conclusions and Future Work}

In this paper, we have addressed an initial study about the relation between the equivalence classes provided by both an attribute reduction and the least local congruence containing such a reduction in FCA. In particular, we have analyzed more in detail the cases when the induced equivalence relation does not provide convex sublattices as equivalence classes and the behavior of the local congruence when we use it in these cases. As a consequence, we have observed that the use of local congruence relations modifies the subset of unconsidered attributes. Moreover, we have stated conditions on the attribute-concepts and the meet-irreducible elements of the concept lattice associated with a context in order to detect when an equivalence class is not a convex sublattice. All ideas presented in this paper have been illustrated by means of different examples.

As future work, we are interested in continuing the study of influence of local congruences in the attribute reduction of a dataset. For example, we will analyze the relationship of the use of local congruences with attribute implications and how the removed attributes can be recovered from the set of attribute implications associated with the context. Furthermore, we will explore the ideas presented in this paper in the fuzzy framework of the multi-adjoint concept lattices.

\section{References}

1. Alcalde, C., Burusco, A.: Study of the relevance of objects and attributes of $L$ fuzzy contexts using overlap indexes. In: Medina, J., et al. (eds.) IPMU 2018. CCIS, vol. 853, pp. 537-548. Springer, Cham (2018). https://doi.org/10.1007/9783-319-91473-2_46

2. Antoni, L., Krajči, S., Krídlo, O.: Constraint heterogeneous concept lattices and concept lattices with heterogeneous hedges. Fuzzy Sets Syst. 303, 21-37 (2016)

3. Aragón, R.G., Medina, J., Ramírez-Poussa, E.: Weaken the congruence notion to reduce concept lattices. Stud. Comput. Intell. 1-7 (2019, in press)

4. Belohlavek, R., Cordero, P., Enciso, M., Mora, A., Vychodil, V.: Automated prover for attribute dependencies in data with grades. Int. J. Approx. Reason. 70, 51-67 (2016)

5. Belohlávek, R., Vychodil, V.: Attribute dependencies for data with grades II. Int. J. Gen Syst 46(1), 66-92 (2017)

6. Benítez-Caballero, M.J., Medina, J., Ramírez-Poussa, E., Ślęzak, D.: A computational procedure for variable selection preserving different initial conditions. Int. J. Comput. Math. 97(1-2), 387-404 (2019)

7. Chen, J., Mi, J., Xie, B., Lin, Y.: A fast attribute reduction method for large formal decision contexts. Int. J. Approx. Reason. 106, 1-17 (2019)

8. Cornejo, M.E., Medina, J., Ramírez-Poussa, E.: Attribute reduction in multiadjoint concept lattices. Inf. Sci. 294, 41-56 (2015) 
9. Cornejo, M.E., Medina, J., Ramírez-Poussa, E.: Computing the validity of attribute implications in multi-adjoint concept lattices. In: International Conference on Computational and Mathematical Methods in Science and Engineering, CMMSE 2016, Vol. II, pp. 414-423 (2016)

10. Cornejo, M.E., Medina, J., Ramírez-Poussa, E.: Attribute and size reduction mechanisms in multi-adjoint concept lattices. J. Comput. Appl. Math. 318, 388-402 (2017). Computational and Mathematical Methods in Science and Engineering CMMSE-2015

11. Cornejo, M.E., Medina, J., Ramírez-Poussa, E.: Characterizing reducts in multiadjoint concept lattices. Inf. Sci. 422, 364-376 (2018)

12. Ganter, B., Wille, R.: Formal Concept Analysis: Mathematical Foundation. Springer, Heidelberg (1999). https://doi.org/10.1007/978-3-642-59830-2

13. Konecny, J., Krajča, P.: On attribute reduction in concept lattices: the polynomial time discernibility matrix-based method becomes the CR-method. Inf. Sci. 491, 48-62 (2019)

14. Li, J., Kumar, C.A., Mei, C., Wang, X.: Comparison of reduction in formal decision contexts. Int. J. Approx. Reason. 80, 100-122 (2017)

15. Liñeiro-Barea, V., Medina, J., Medina-Bulo, I.: Generating fuzzy attribute rules via fuzzy formal concept analysis. In: Kóczy, L.T., Medina, J. (eds.) Interactions Between Computational Intelligence and Mathematics. SCI, vol. 758, pp. 105-119. Springer, Cham (2018). https://doi.org/10.1007/978-3-319-74681-4_7

16. Medina, J.: Relating attribute reduction in formal, object-oriented and propertyoriented concept lattices. Comput. Math. Appl. 64(6), 1992-2002 (2012)

17. Ren, R., Wei, L.: The attribute reductions of three-way concept lattices. Know.Based Syst. 99(C), 92-102 (2016)

18. Shao, M.-W., Li, K.-W.: Attribute reduction in generalized one-sided formal contexts. Inf. Sci. 378, 317-327 (2017)

19. Vychodil, V.: Computing sets of graded attribute implications with witnessed nonredundancy. Inf. Sci. 351, 90-100 (2016) 